\title{
The utility of contrast-enhanced ultrasonography in diagnosing child's vesicoureteral reflux - a case report and a review of the literature
}

\author{
Maria Oana Marginean', Carmen Duicu ${ }^{1,2}$, Vladut Sasaran', Cristina Oana Marginean ${ }^{1,2}$, \\ Lorena Elena Melit ${ }^{1,2}$ \\ ${ }^{1}$ University of Medicine and Pharmacy, Targu Mures, Romania \\ ${ }^{2}$ Pediatrics Clinic 1, Targu Mures, Romania
}

\begin{abstract}
Vesicoureteral reflux represents the reflow of bladder content in the ureter and pelvicalyceal system with their subsequent dilation. Most of the cases of urinary tract infections in child are associated with vesicoureteral reflux. We present the case of a 3-year-old female patient, with a history of multiple symptomatic urinary tract infections, who was admitted in our clinic for specialty investigations and establishment of an adequate therapy. The clinical exam and the laboratory tests did not point out any pathological elements. The native abdominal ultrasound was without pathological modifications, while the contrast-enhanced ultrasonography revealed bilateral vesicoureteral reflux. The renal scintigraphy with DMSA showed impaired renal function on the right side, of $39 \%$ with duplicity of the collector system and moderate dilation of the superior pelvis, and normal function of the left kidney. The recommendations consisted in long-term antibiotic prophylaxis, contrast-enhanced ultrasonography once a year and nephrologist consult every 6 months. The particularity of the case consists in diagnosing a bilateral vesicoureteral reflux by contrast-enhanced ultrasonography in a female patient with a history of multiple urinary tract infections, in whom the native renal ultrasound did not point out any pathological elements.
\end{abstract}

Keywords: urinary tract infection, vesicoureteral reflux, SonoVue ${ }^{\circledR}$, child

\section{INTRODUCTION}

Vesicoureteral reflux represents the reflow of the bladder content in the ureter and pelvicalyceal system with their subsequent dilation. One of the major complication that can appear in case of patients diagnosed with vesicoureteral reflux is represented by the recurrence of symptomatic urinary tract infections. In fact, most of the times, the diagnosis of vesicoureteral reflux and of subsequent hydronephrosis is a consequence of routine evaluation in case of a symptomatic urinary tract infection. These repeated infections will lead to the development of renal scars, and small children are considered particularly vulnerable to develop this type of renal sequels and their complications (1). Thus, in United Kingdom, all children under the age of 5 years must be investigated after the first confirmed episode of urinary tract infection $(2,3)$. The imagistic methods are usually used in order to establish the diagnosis of vesicoureteral reflux. Traditionally, voiding cystourethrography is the most frequent imagistic diagnostic tool used in order to point out the vesicoureteral reflux, but it disadvantage is represented by radiation exposure, unwanted effect especially in children. Thus, the more recent guidelines do not recommend usually to perform voiding cystourethrography after the first episode of urinary tract infection associated with fever. Therefore, in order to eliminate this unwanted effect, on the middle of ' $90 \mathrm{~s}$, it was proposed the contrastenhanced renal ultrasonography as being a radiation-free method, useful for the detection and monitoring of vesicoureteral reflux in children (4). First generation contrast agents contain stabilized microbubbles filled with air, among which Levovist ${ }^{\circledR}$ was the only one approved for use in pediatrics in a few of the European countries (5). After approximately 10 years, this agents was replaced by second generation contrast agents. Thus, SonoVue ${ }^{\circledR}$ is 
a second generation contrast agent that contains stabilized microbubbles filled with gas and it was released on the market at the end of $2001(6,7)$. The method consists initially in the introduction of a urinary catheter in the bladder in order to administer afterwards the contrast agent in the bladder followed by ultrasonographic monitoring. Thus, the reflow of microbubbles in the ureter and pelvicalyceal system can be detected by ultrasonography. Depending on the ultrasonographic aspect of the ureter and pelvicalyceal system by measuring their diameters, it will be established the diagnosis of vesicoureteral reflux and the presence of hydronephrosis, being possible to estimate their degree.

\section{CASE PRESENTATION}

We present the case of a 3-year-old female patient, who was admitted in our clinic with the suspicion of a vesicoureteral reflux for specialty investigations and establishment of an adequate therapy. Her personal history revealed 4 episodes of acute cystitis treated ambulatory and 3 episodes of symptomatic high urinary tract infection for which the patient was admitted in the regional hospital receiving antibiotics by vein, the last episode approximately 3 months before the admission in our clinic, since when she was receiving antibiotic prophylaxis (Augmentin).

The clinical exam on the moment of admission was without pathological elements, weight $12.5 \mathrm{~kg}$. All laboratory tests were in normal ranges. We also performed native abdominal ultrasound that did not reveal any pathological modifications. Therefore, we decided to perform contrast-enhanced abdominal ultrasound with a second generation contrast agent - SonoVue $\AA$. After introducing a urinary catheter into the bladder, we introduced on the catheter the contrast agent. Firstly, the ultrasonography revealed the complete fulfil of the bladder, and afterwards we detected the ureters bilaterally, more dilated on the right side with a diameter of approximately 6-7 $\mathrm{mm}$ in comparison to the left side where the diameter was of approximately 4-5 mm (Fig. 1).

Therefore, we depicted bilateral vesicoureteral reflux. Next, on the right side, we noticed the complete opacification of the pelvicalyceal system, with a dilated pelvis, measuring approximately 18.5/10 mm (Fig. 2).

On the left side, the contrast-enhanced ultrasonography revealed the complete opacification of the pelvicalyceal system but with milder dilations comparatively to the right kidney, of approximately $10 / 7 \mathrm{~mm}$. In conclusion, the contrast-enhanced ultrasonography revealed bilateral vesicoureteral reflux, estimated to be of $3^{\text {rd }}$ degree on the right side, and $2^{\text {nd }}$ degree on the left side, respectively. In order to assess the renal function, we performed also a renal scintigraphy with dimercaptosuccinic acid (DMSA), which showed right renal hypofunction, of $39 \%$ with duplicity of the collector system and moderate dilation of the superior pelvis. The left kidney presented normal function, of $61 \%$. Also, the right kidney was smaller in size comparatively to the left one, $62 \mathrm{~mm}$ in comparison to $71 \mathrm{~mm}$. The scintigraphy with DMSA pointed out also areas of decreases uptake in the upper third of the right kidney, without revealing any renal scars.

Based on all mentioned above, we established the diagnoses of bilateral vesicoureteral reflux and reflux nephropathy of the right kidney. The recom-

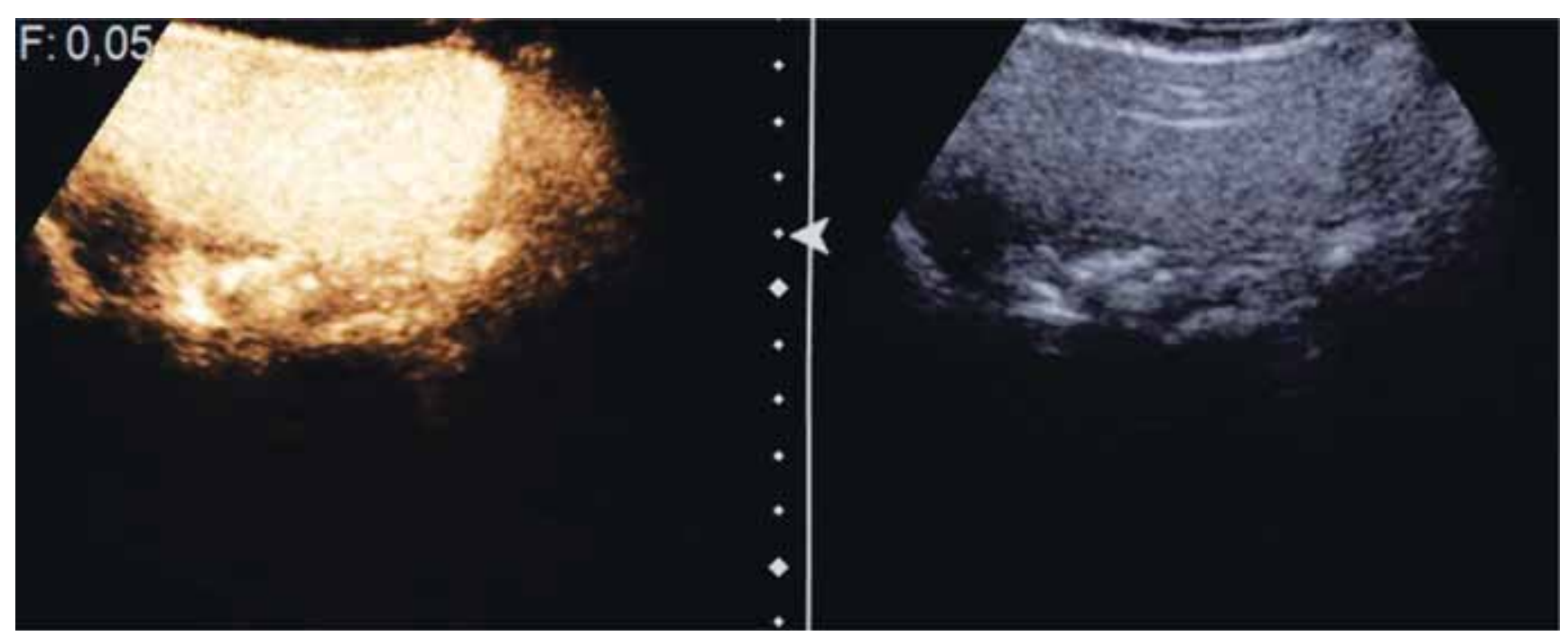

FIGURE 1. Aspect of the bladder after contrast administration revealing bilateral vesicoureteral reflux 


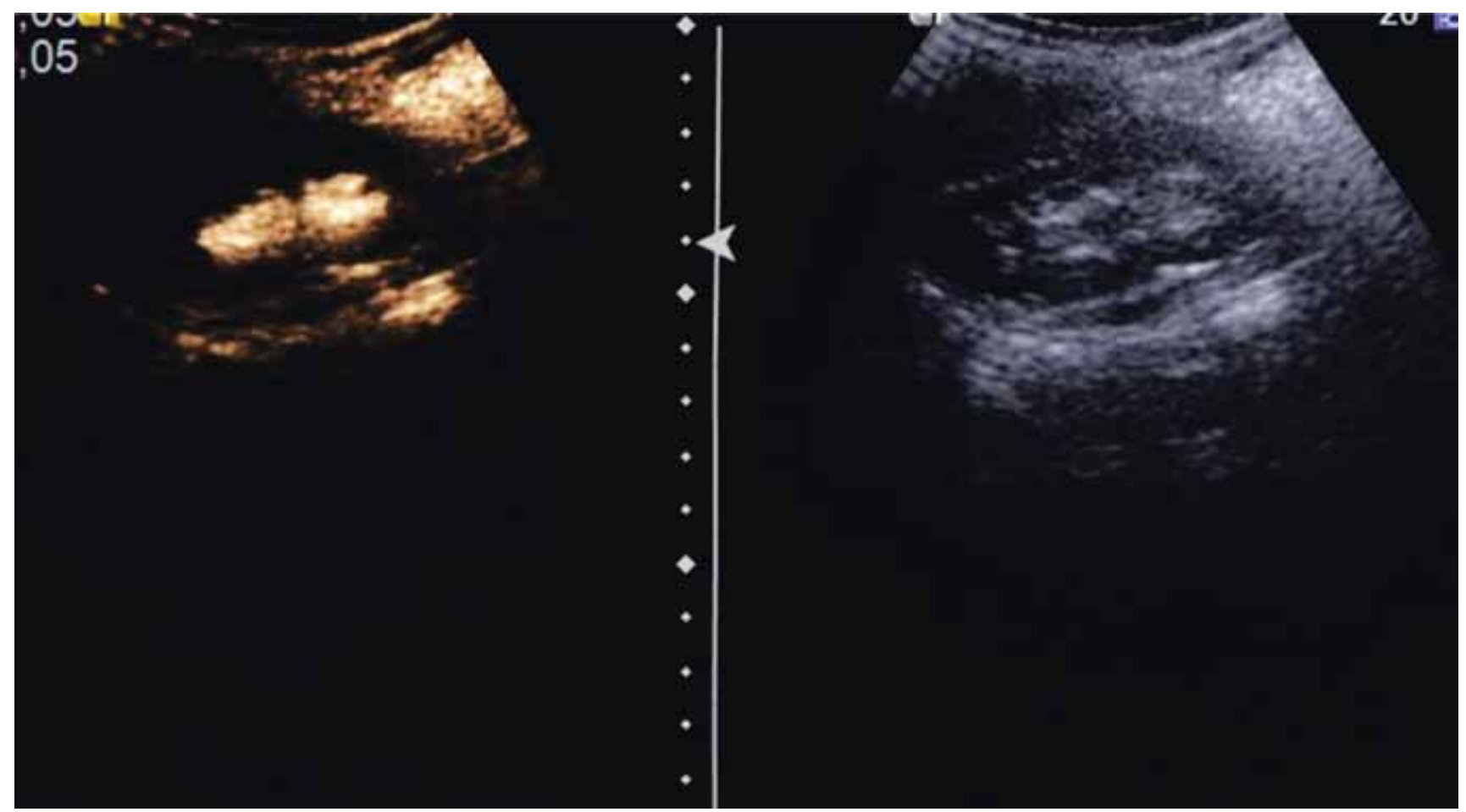

FIGURE 2. Right kidney - aspect after contrast administration

mendations at discharge consisted in the continuation of antibiotic prophylaxis with Augmentin suspension a $400 \mathrm{mg} / 57 \mathrm{mg} / 5 \mathrm{ml}, 1.5 \mathrm{ml}$ every evening for a month, followed by sulfamethoxazole suspension a $40 \mathrm{mg} / 5 \mathrm{ml}, 3 \mathrm{ml}$ every evening in the next month, and in the third month Cephalexin suspension a $125 \mathrm{mg} / 5 \mathrm{ml}, 5 \mathrm{ml}$ every evening, with longterm regimen repeating. Also, we recommended periodic reevaluation of the vesicoureteral reflux by contrast-enhanced ultrasonography once a year, nephrologist consult every 6 month, or earlier in case of fever or symptoms suggesting a urinary tract infection.

The particularity of the case consists in diagnosing a bilateral vesicoureteral reflux by contrast-enhanced ultrasonography in a female patient with a history of multiple urinary tract infections, in whom the native renal ultrasound did not point out any pathological elements.

\section{DISCUSSIONS}

Urinary tract infections represent a pathology with increased frequency in pediatric age, and one of the most frequently encountered factors involved in their pathogenesis is vesicoureteral reflux, being considered to be associated in most of the cases with urinary tract infection (8). Vesicoureteral reflux is associated in approximately $25-40 \%$ of the cases with urinary tract infections in children, with- out significant differences regarding gender impairment, except during infancy (9). During this period, females are more frequently affected. Thus, it has been proven that during infancy, vesicoureteral reflux is present in approximately $30 \%$ of the boys and $43 \%$ of the girls that present with symptomatic urinary tract infection (10). The case presented by us also involves a female, even though not during infancy, describing a 3-year-old female patient. Vesicoureteral reflux is not related only to the abnormal length of the intravesical part of the ureter, but it also represents a dysfunctional issue, case in which the patients can also present bladder emptying problems or bowel dysfunctions (11). Nevertheless, there are also cases in which the vesical dysfunction can be the cause of vesicoureteral reflux. The patient presented above did not present any of these associations. Even though multiple studies from the past considered the vesicoureteral reflux is always associated with reflux nephropathy and renal scarring (12), it seems that recently, there are contradictory data regarding both the role of vesicoureteral reflux in the development of urinary tract infection (13), and the effect of antibiotic prophylaxis in preventing pyelonephritis and renal scarring (14). Nevertheless, in case of our patient, we diagnosed both bilateral vesicoureteral reflux and nephropathy reflux of the right kidney.

The most frequently used methods are voiding cystourethrography for the detection of vesi- 
coureteral reflux and renal scintigraphy with DMSA for infection localization and the detection or prediction of potential renal scars. The major disadvantages of these two methods are represented by patient's exposure to radiations and the minimal invasiveness they involve. Nevertheless, the irradiation is much more reduced in case of DMSA scan, tending to extend its use over the voiding cystourethrography. Thus, in order to eliminate these two inconvenient, but also due to the fact that this pathology presents an increased frequency in children, the contrast-enhanced ultrasonography was introduced. Initially, there were used first generation contrast agents, but nowadays second generation contrast agents are available on the marker, such as SonoVue ${ }^{\circledR}$, which due to the gas contained in the microbubbles can be used also for the assessment of the urinary system, these microbubbles being detected by ultrasonography also in the urinary tract (15). Also, due to the more decreased fragility of microbubbles contained by second generation contrast agents, the latter present a longer detection length by ultrasonography (15). Therefore, SonoVue ${ }^{\circledR}$ was assessed in multiple recent studies. Thus, according to a survey study which involved multiple radiology institutions from Europa, approximately 4,131 children underwent contrast-enhanced ultrasonography by administration of SonoVue ${ }^{\circledR}$ inside the bladder, without any side effect due to the administration of this contrast agent (16). Nevertheless, there are certain symptoms that were reported by the patients after this procedure, life dysuria, urinary retention, transitory abdominal pain, increased frequency of micturition, blood or mucus discharge, anxiety or crying during micturition, vomiting, perineal irritation or urinary tract infection (4). This symptoms are not only relatively rare, but they were also described in case of the other imagistic procedures used for the diagnosis of vesicoureteral reflux, and are most probably due to the catheterization of the bladder not being related to the administration of contrast agents.

The method of contrast-enhanced ultrasonography for depicting vesicoureteral reflux is superior to traditional imagistic methods, being a minimum invasive procedure, without involving any risk of irradiation, but in comparison to the voiding cystourethrography, it can not show exactly the degree of the vesicoureteral reflux. Nevertheless, the main limitation of this method is represented by the fact that we can't see simultaneously the urethra and the reno-ureteral system in comparison to the traditional imagistic methods. Also, the association of this method with an increased risk of catheterization associated urinary tract infection is another disadvantage that was reported to be up to $80 \%$ in case of short-term catheterization (17-19).

Most guidelines recommend as a first choice the voiding cystourethrography for establishing the degree of vesicoureteral reflux, voiding contrast-enhanced ultrasonography being the method recommended for monitoring the reflux. Nevertheless, the case presented above might suggest the use of voiding contrast-enhanced ultrasonography as a first choice in patients with repeated urinary tract infections, an alternative to contrast voiding cystourethrography, but without being a mandatory recommendation.

\section{CONCLUSIONS}

Contrast-enhanced ultrasonography using second generation contrast agents for the diagnosis of vesicoureteral reflux represents a relatively easy procedure, effective, non-invasive, without irradiation. This method is very useful in case of pediatric patients who present repeated urinary tract infections.

\section{REFERENCES}

1. Vernon S.J., Coulthard M.G., Lambert H.J., Keir M.J., Matthews J.N. New renal scarring in children who at age 3 and 4 years had had normal scans with dimercaptosuccinic acid: follow up study. Bmj 1997; 315:905-908.

2. Working Group of the Research Unit of the Royal College of Physicians. Guidelines for the management of acute urinary tract infection in childhood. J R Coll Physicians 1991;25:36-43.

3. UTI (lower) - acute Prodigy [http://www.prodigy.nhs.uk/guidance.asp?gt=UTI\%20 $-\% 20$ children].

4. Papadopoulou F,, Ntoulia A,, Siomou E,, Darge K. Contrast-enhanced voiding urosonography with intravesical administration of a second-generation ultrasound contrast agent for diagnosis of vesicoureteral reflux: prospective evaluation of contrast safety in 1,010 children. Pediatr Radiol 2014; 44(6):719-28.

5. Schlief R. Developments in echo-enhancing agents. Clin Radiol 1996; 51:5-7.

6. Schneider M., Arditi M., Barrau M.B. et al. BR1: a new ultrasonographic contrast agent based on sulfur hexafluoride-filled microbubbles. Invest Radiol 1995;30:451-457.

7. Schneider M. SonoVue, a new ultrasound contrast agent. Eur Radiol 1999; 9:S347-S348

8. Tse K.S., Wong L.S., Lau H.Y. Paediatric vesicoureteric reflux imaging: where are we? Novel ultrasound-based voiding urosonography. Hong Kong Med J 2014; 20:437-443.

9. Sargent M.A. What is the normal prevalence of vesicoureteric reflux? Pediatr Radiol 2000; 30:587-593.

10. Fong K.W., Wong S.N. Symptomatic urinary tract infection in children: experience in a regional hospital in Hong Kong. Hong Kong J Paediatr 2004; 9:30-36.

11. Koff S.A., Wagner T.T., Jayanthi V.R. The relationship among dysfunctional elimination syndromes, primary vesicoureteral reflux and urinary tract infections in children. J Urol 1998; 160:1019-1022.

12. Bailey R.R. The relationship of vesico-ureteric reflux to urinary tract infection and chronic pyelonephritis-reflux nephropathy. Clin Nephrol 1973; 1:132-141. 
13. Ditchfield M.R., De Campo J.F., Cook D.J., et al. Vesicoureteral reflux: an accurate predictor of acute pyelonephritis in childhood urinary tract infection? Radiology 1994; 190:413-415

14. Pennesi M., Travan L., Peratoner L., et al. North East Italy Prophylaxis in VUR study group. Is antibiotics prophylaxis in children with vesicoureteral reflux effective in preventing pyelonephritis and renal scars? A randomized, controlled trial. Pediatrics 2008; 121:e1489-1494.

15. Spinazzi A. Emerging clinical applications for contrast-enhanced ultrasonography. Eur Radiol 2001; 11(suppl3):E7-E12.

16. Riccabona M. Application of a second generation US contrast agent in infants and children-a European questionnaire-based survey. Pediatr Radiol 2012; 42:14711480.
17. Parker D., Callan L., Harwood J., Thompson D.L., Wilde M., Gray M. Nursing interventions to reduce the risk of catheter-associated urinary tract infection. Part 1: catheter selection. J Wound Ostomy Continence Nurs 2009; 36:23-34. doi:.10.1097/01.WON.0000345173.05376.3e.

18. Maki D.G., Tambyah P.A. Engineering out the risk for infection with urinary catheters. Emerg Infect Dis 2001; 7:342-347. doi:.10.3201/eid0702.700342

19. Willson M., Wilde M., Webb M.L. et al. Nursing interventions to reduce the risk of catheter-associated urinary tract infection: Part 2: staff education, monitoring, and care techniques. J Wound Ostomy Continence Nurs 2009; 36:137-154.

Conflict of interest: none declared Financial support: none declared 